\title{
La reconstruction de l'église de Lambézellec (Finistère) sous le Second Empire
}

\section{Louis Chauris}

\section{(C) OpenEdition \\ Journals}

Édition électronique

URL : http://journals.openedition.org/abpo/1287

DOI : $10.4000 / a b p o .1287$

ISBN : 978-2-7535-1494-2

ISSN : 2108-6443

\section{Éditeur}

Presses universitaires de Rennes

\section{Édition imprimée}

Date de publication : 20 juillet 2004

Pagination : 119-144

ISBN : 978-2-7535-0031-0

ISSN : 0399-0826

\section{Référence électronique}

Louis Chauris, "La reconstruction de l'église de Lambézellec (Finistère) sous le Second Empire ",

Annales de Bretagne et des Pays de l'Ouest [En ligne], 111-2 | 2004, mis en ligne le 20 juillet 2006, consulté le 21 avril 2019. URL : http://journals.openedition.org/abpo/1287 ; DOI : 10.4000/abpo.1287

\section{(C) Presses universitaires de Rennes}




\title{
La reconstruction de l'église de Lambézellec (Finistère) sous le Second Empire
}

\author{
Louis CHAURIS \\ directeur de recherche au CNRS (e.r.)
}

Dans de nombreuses communes du diocèse de Quimper et de Léon, comme ailleurs en Bretagne, la reconstruction de l'église paroissiale a été entreprise au cours de la seconde partie du XIX ${ }^{\mathrm{e}}$ siècle $^{1}$. La frénésie qui poussait curés et recteurs à rebâtir les lieux de culte était telle que l'on avait qualifié cette obsession de " maladie de la pierre ${ }^{2}$ ". Emportés par leur zèle, certains pasteurs désiraient même édifier " de petites cathédrales " ce qui pouvait susciter sourires et critiques. Cette volonté de la nouveauté à tout prix se fondait toutefois, bien souvent, sur deux motifs plus sérieux : sous les injures du temps, la vieille église risquait de s'écrouler; avec l'augmentation des fidèles, l'édifice s'avérait notoirement insuffisant. À ce désir du clergé, appuyé par les souhaits des conseils municipaux, venait aussi s'adjoindre l'ambition des architectes consultés, heureux tout à la fois d'exprimer leur savoir-faire d'une empreinte durable et, ipso facto, d'obtenir de substantiels honoraires.

Ainsi, les motivations qui ont entraîné la destruction de bien des églises anciennes s'avèrent fort complexes, à tel point qu'il est quelquefois difficile de préciser si la reconstruction totale était une meilleure solution que la restauration partielle. C'est qu'en effet plusieurs possibilités étaient $a$ priori envisageables. La moins onéreuse était de restaurer l'ancien édifice, tout en l'agrandissant : cette option a été retenue à Plouvorn ${ }^{3}$. Conserver seulement le clocher, comme à Plouneour-Trez ${ }^{4}$, voire à la fois le clocher et le porche méridional, comme à Landivisiau. Démolir l'ancienne église et

1. Couffon, René et LE BARS, Alfred, Diocèse de Quimper et de Léon, Nouveau répertoire des églises et chapelles, Quimper, 1988, $552 \mathrm{p}$.

2. FEUTREN, Jean, Bulletins paroissiaux, regroupés en trois forts volumes, intitulés " Autour de Pleyber-Christ".

3. Arch. dép. du Finistère, 1 V 488. Le clocher avait été démonté en 1842 et remonté quelques années plus tard; l'église, agrandie ultérieurement.

4. Arch. dép. du Finistère, 1 V 481. 
en rebâtir une autre à son emplacement même, comme à Plouénan ${ }^{5}$. Laisser la vieille église à son destin et en élever une autre à proximité, cas de Henvic $^{6}$. Ou encore, remployer, lors du déplacement de l'église, les pierres du vieux bâtiment, comme dans le cas de Lochrist-Le Conquet ${ }^{7}$ et de SaintHouardon à Landerneau ${ }^{8}$...

Il a paru digne d'intérêt d'expliciter les modalités de la reconstruction d'une église sous le Second Empire par un exemple précis, pour lequel les données archivistiques sont abondantes, et l'édifice encore en partie conservé dans son état initial. Dans cette perspective, a été retenue l'église de Lambézellec, aujourd'hui dans la ville de Brest, mais qui fut, longtemps, le lieu de culte d'une importante commune. La richesse des archives permet, en effet, d'envisager en détail les multiples facettes des problèmes soulevés, tant humains que techniques et financiers... souvent inextricablement entremêlés. Ce faisant, sera éclairée une page d'histoire locale qui, bien que liée à un seul édifice religieux, s'articule également à la vie communale, mieux, à l'activité économique de la contrée sous le Second Empire et, par suite, dépasse largement, semble-t-il, un thème qui, au premier abord, pouvait paraître de portée limitée.

L'actuelle église de Lambézellec, dédiée à saint Laurent, est un édifice en forme de croix latine, offrant une nef de sept travées avec bas-côtés, un transept et un chœur de neuf arcades, entouré d'un déambulatoire. De chaque côté des collatéraux s'ouvre un porche au droit de la troisième travée de la nef. Le clocher, détruit lors de la dernière guerre, présentait une double galerie et une flèche octogonale avec quatre clochetons d'angle ${ }^{9}$. Cet édifice construit de 1863 à 1865, restauré après la guerre ${ }^{10}$, remplace l'église paroissiale qui remontait seulement à 1750. Mais ce bâtiment avait été bâti " dans des conditions si malheureuses ", qu'il fallait songer à le remplacer un siècle plus tard. À son tour, l'église de la seconde partie moitié du XVIII ${ }^{\mathrm{e}}$ siècle succédait à un édifice sur lequel " nous ne savons guère que ce que nous apprend le procès-verbal des prééminences dressé peu avant sa démolition en $1750^{11}$ ". Il était alors constaté que l'église était " en quelque sorte pavée d'environ 110 tombes placées sur 10 rangées " et qu'à l'extérieur, il y avait une lisière portant de nombreux écussons. Au total, fort peu de données...

5. Arch. dép. du Finistère 1 V 462 et arch. évêché Quimper (Plouénan), 1884-1886; cependant, le beffroi et la flèche ne devaient être exécutés qu'au tout début du Xx ${ }^{\mathrm{e}}$ siècle.

6. Henvic (1899-1903). Arch. dép. du Finistère, 1 V 359.

7. Le Conquet. Arch. dép. du Finistère, 1 V 320 et arch. de l'évêché de Quimper, 1855-1858.

8. Landerneau-Saint-Houardon (achevé en 1861). Arch. de l'évêché de Quimper.

9. Couffon, R. et LE BARs, Alfred, op. cit. Voir aussi les plans conservés aux archives de l'évêché de Quimper.

10. Érection d'une flèche en béton. Le nouveau maître-autel a été consacré le 24 janvier 1952.

11. Peyron, Paul et Abgrall, Jean-Marie, Notices sur les paroisses, Bull. diocésain d'Histoire et d'Archéologie, vol. V, 1919, p. 171-172. 


\section{Pour une restauration...}

Le 18 octobre 1854, le conseil de fabrique de l'église paroissiale de Lambézellec se réunit en séance extraordinaire pour entendre son président lui soumettre un plan et un devis de restauration de ladite église ${ }^{12}$. Le président souligne qu'« il est de notoriété publique qu'une amélioration est depuis longtemps indispensable ». Il constate qu'une partie de la population déserte l'édifice, n’y trouvant "ni place commode, ni sécurité pour la santé ". Plus grave, " ses murs tombent en ruines, sa toiture est ouverte à toutes les pluies ". Et dans une envolée, il va jusqu'à déclarer : "La crèche de Bethléem n'était pas plus pauvre que notre édifice. "

Le devis de restauration, soumis à l'examen du conseil, s'élève à 26171,29 F. Plans et devis ont été dressés par M. Jugelet, architecte de l'arrondissement de Brest. Le président regrette de ne pouvoir présenter le plan d'une nouvelle église, " plus digne de la majesté du culte [et] de l'importance de la commune ». Et il ajoute : "Hélas, l'administration municipale ne peut plus songer à réaliser ses beaux projets votés le 8 décembre 1853. " Mais ne pourrait-on pas attendre que " la commune soit à même de rebâtir une église plus belle et plus spacieuse "? En fait, comme remarque le président, "ce cri " a été répété depuis plus de vingt ans, avec le seul résultat d'ajourner toute réparation - ce qui a conduit à l'actuel état de délabrement de l'édifice. D'ailleurs, l'administration municipale « n'ayant rien fait pour l'église dans le temps de sa longue prospérité, il serait déraisonnable de compter sur elle lorsqu'elle ne peut pas payer ses dépenses ordinaires".

Dans ces conditions, la fabrique ne doit compter que sur ses ressources qui s'élèvent actuellement à $10170 \mathrm{~F}$, se répartissant ainsi : une somme disponible $(4000 \mathrm{~F})$, à laquelle s'ajoute la vente d'immeubles $(4670 \mathrm{~F})$ et des dons volontaires (1500 F). Pour combler le déficit de $16000 \mathrm{~F}$, le président propose deux solutions : d'une part, contracter un emprunt gratuit de $10000 \mathrm{~F}^{13}$; d'autre part, demander les $6000 \mathrm{~F}$ restant « à la pieuse générosité de [la] population qui... n'attend qu'un commencement d'exécution pour souscrire à l'œuvre dont elle comprend toute l'urgence ». Quelques secours sont aussi espérés du gouvernement, du département ou de la commune. Le conseil sollicite également " la puissante protection de $\mathrm{M}^{\mathrm{gr}}$ l'Évêque de Quimper et de Léon qui sait que la paroisse de Lambézellec, l'une des plus importantes du diocèse, est la plus pauvre en fait d'église ".

À première vue, le problème de la place disponible pour les fidèles dans l'église de Lambézellec paraît dramatique : la commune compte 12000 âmes. Mais comme le fait remarquer le président de la fabrique lors de la même séance du 18 octobre 1854, rarement l'édifice recevra plus de

12. Arch. dép. du Finistère, 1 V 376.

13. Le curé, $3000 \mathrm{~F}$ et sept membres du conseil, $1000 \mathrm{~F}$ chacun, s'engageant à épurer gratuitement les $10000 \mathrm{~F}$, remboursables sans intérêt quand les économies de la fabrique le permettront. 
2300 personnes. Les raisons de cette énorme différence sont multiples : les problèmes de distance, mais aussi de langage (breton-français) et également les habitudes " qui éloigneront toujours du bourg... une grande partie de la population qui est plus voisine de Brest".

Le compte rendu des délibérations du conseil de fabrique que nous venons de citer longuement, conduit, dès à présent, à formuler quelques observations :

- l'état de délabrement de l'église ne cesse de s'aggraver avec le report incessant des réparations indispensables;

- la générosité des membres du conseil de fabrique qui acceptent l'emprunt gratuit dont le remboursement risque d'être très long, et aussi des fidèles de la paroisse, se doit d'être soulignée;

- les difficultés financières de la fabrique ne peuvent être compensées par le conseil municipal qui a peine à faire face aux dépenses ordinaires.

En fait, le dilemme suivant se pose à Lambézellec : restaurer l'édifice ou le reconstruire. À l'évidence, la première solution est, financièrement, et de loin, la moins coûteuse. Et pourtant, les ressources de la fabrique ne peuvent y pourvoir.

Comme nous allons le constater, les problèmes de financement apparaîtront sous des formes diverses, tout au long du projet de la reconstruction de l'église de Lambézellec, solution qui, finalement, allait prévaloir.

\section{Pour une reconstruction totale}

Le projet de restauration de l'église, élaboré à l'instigation du conseil de fabrique, soulève des critiques qui inspirent au curé un autre projet : le chœur, la sacristie et les autels collatéraux seront conservés, tandis que la nef et les bas-côtés seront allongés; quant au clocher, il sera démonté pierre par pierre et rétabli à la fin des travaux.

Mais voici qu'à son tour, le maire présente un autre point de vue devant le conseil municipal, lors de la séance du 11 novembre $1855^{14}$ : il ne s'agit plus d'agrandir l'édifice, mais de le reconstruire entièrement. Lors de la délibération, l'un des membres du conseil déclare qu'il serait choquant que la " maison du Seigneur seule ne fut remarquable que par son misérable aspect ". Il ajoute que si les églises doivent, avant tout, satisfaire aux besoins du culte, elles sont aussi des œuvres municipales et " sous ce rapport, imposent au pouvoir temporel des obligations ". Puis, il s'élève, vivement contre la proposition suggérée par M. le curé, qualifiée d'« idée on ne peut plus malheureuse ". Son argumentation est très simple : pourquoi abattre les murs latéraux, déplacer le clocher, pour n'obtenir qu'une " église, moitié vieille, moitié neuve, et faite de pièces et de morceaux ", le tout pour une dépense considérable qu' "il ne faudrait pas beaucoup augmenter pour édifier une église neuve, s'harmonisant dans toutes ses parties "?

14. Arch. dép. du Finistère, 1 V 376. 
La décision de reconstruire l'église étant prise, le conseil municipal émet le curieux projet d'ouvrir un concours pour la présentation des plans et devis du futur édifice. Dans une lettre du 11 janvier $1858^{15}$, l'administration préfectorale se demande toutefois s'il est bien nécessaire de recourir à un moyen exceptionnel et coûteux pour une construction qui ne doit " rien avoir d'extraordinaire ». Les ressources de la commune de Lambézellec s'opposent à ce qu'elle puisse songer "à faire élever une église véritablement monumentale et hors ligne ". Dans ces conditions, il est évident que l'on trouvera dans le pays un architecte capable de dresser un projet d'église. Par ailleurs, le conseil municipal a également exprimé l'idée de confier la direction et la surveillance des travaux " à un architecte autre que l'auteur du projet ". Une telle proposition s'avère tout à fait irréaliste : "Comment penser en effet que l'architecte dont le projet aura été admis, consentira jamais à confier l'exécution à un homme qu'il ne connaîtra peutêtre pas ou qui n'aura pas sa confiance. Il y consentira d'autant moins que la plus forte part de la rémunération serait pour ce dernier, d'après le système du conseil municipal. "

Cet étonnant projet devait être rapidement abandonné. Dans sa séance du 29 mai 1858, le conseil municipal renonce à son idée de concours et charge l'architecte diocésain de faire un plan en rapport avec les besoins de la commune. Lors de sa délibération du 5 septembre 1858, le conseil municipal adopte plans et devis qui ont été dressés par l'architecte diocésain Joseph Bigot, sous réserve de modifications de détail; il sera facultatif d'ajourner le dallage et le clocher ou, au contraire, d'achever immédiatement ces travaux selon les ressources alors disponibles. La dépense totale est fixée à la somme de 156467,69 F. Le 20 octobre de la même année, le sous-préfet fait part au préfet de son avis sur les plans et devis de J. Bigot : le projet est qualifié de " fort bien conçu et tout à fait en rapport avec les besoins et les exigences d'une commune aussi importante ${ }^{16}$ ".

Dans une longue lettre au préfet, datée du 28 février 1861, l'architecte J. Bigot justifie son projet de reconstruction de l'église de Lambézellec et tout particulièrement les dimensions retenues ${ }^{17}$. L'architecte s'efforce de démontrer que l'annexion d'une partie de la commune de Lambézellec par la ville de Brest ${ }^{18}$ ne saurait conduire à diminuer la surface à donner à

15. Ibid.

16. Sur la vie et l'œuvre de Bigot, se reporter à BAYLÉ, Jeanne, "L'architecte quimpérois Joseph Bigot (1807-1894) ", Bulletin de la société archéologique du Finistère, 1977, t. CV, p. 219-277. Architecte départemental du Finistère en 1835, architecte diocésain en 1837, Bigot était un " travailleur acharné ", ne connaissant "ni vacances, ni dimanches, ni jours fériés ". Parmi ses œuvres, extrêmement nombreuses, citons les flèches de la cathédrale de Quimper, le château de Keriolet à Concarneau et surtout 27 églises (3 sous LouisPhilippe, 14 sous le Second Empire, 8 entre 1870 et 1878, enfin 2 en 1886). La première église est celle de Baye (1842), la dernière, celle de Saint-Thurien (1886); Lambézellec (1863-1865) occupe ainsi une position centrale. Après Douarnenez $\left(870 \mathrm{~m}^{2}\right)$ et Saint-Houardon de Landerneau $\left(860 \mathrm{~m}^{2}\right)$, Lambézellec $\left(826 \mathrm{~m}^{2}\right)$ est la plus vaste église édifiée par Bigot.

17. Arch. dép. du Finistère, 1 V 376.

18. Opération qui sera réalisée au début des années 1860. 
l'église. Et ceci parce que, en 1861, la population de Lambézellec se divise en deux sections principales : l'une est située sur les glacis près des remparts de Brest; ses habitants se rendent rarement aux offices à Lambézellec, étant beaucoup plus proches des églises brestoises; l'autre dans le centre et les environs du bourg. En conséquence, si avant l'annexion, l'église paroissiale de Lambézellec est trop petite, elle le restera aussi après l'annexion. En fait, et l'architecte insiste sur ce point, l'importance de Brest va bientôt s'accroître considérablement avec l'achèvement de son port de commerce et l'arrivée du chemin de fer... Or Lambézellec est avant tout " la banlieue " de ce grand port - d'où sa conclusion : " Le plan de l'église est vaste, comparativement à l'église actuelle, mais je ne pense pas qu'il puisse être réduit sans laisser de stériles regrets. " Et l'architecte d'ajouter : "Une grande église exige un caractère sévère, il est vrai, mais il ne faut pas la rendre aussi nue qu'un forum, par cela même qu'elle se revêt d'un caractère sacré. "

\section{Conseil municipal contre conseil de fabrique}

Comme le cas s'est présenté fréquemment, lors des restaurations ou reconstructions d'églises ${ }^{19}$, des désaccords vont se manifester à Lambézellec entre le conseil de fabrique (et singulièrement le curé) et le conseil municipal (en la personne du maire), sous les regards de l'administration préfectorale.

Suite à l'adoption du projet de Joseph Bigot, le conseil municipal a voté un emprunt de $100000 \mathrm{~F}$ pour couvrir une partie de la dépense, la construction du clocher devant toutefois être alors ajournée. Cependant, ainsi que l'écrit le sous-préfet au préfet, le 20 octobre $1858^{20}$, le conseil municipal n'a pu exprimer d'une manière précise la demande de secours qu'il est dans son intention de formuler, " attendu qu'il ne connaît pas encore dans quelle proportion la fabrique voudra contribuer à la dépense ". Aussi, le sous-préfet demande de « faire saisir la fabrique du projet par l'intermédiaire de l'autorité diocésaine afin qu'elle délibère et qu'elle indique les ressources qu'elle entend affecter à la dépense ".

En fait, la regrettable indécision dans laquelle le conseil de fabrique laisse le conseil municipal sur sa participation, remonte à une date antérieure, comme le laisse clairement entendre une lettre du maire au curé en date du 29 juin $1858^{21}$. Après avoir rappelé que l'administration municipale " prenant au sérieux sa mission et ses traditions ", a jugé de son devoir de poursuivre "l'œuvre tant et si universellement reconnue de la reconstruction de l'église ", le maire fait part de l'appui du sous-préfet estimant qu'il convient de ne laisser " aucun motif aux divisions qui ont reculé d'une manière fâcheuse l'exécution d'une entreprise reconnue urgente ". Aussi, le conseil

19. Parmi les exemples de désaccord entre conseil de fabrique et conseil municipal, citons Saint-Thomas à Landerneau (Arch. dép. du Finistère, 1 V 383), Plounéour-Trez (Arch. dép. du Finistère, 1 V 481), Plouénan (Arch. dép. du Finistère, 1 V 462)...

20. Arch. dép. du Finistère, 1 V 376.

21. Idem. 
municipal se dit " mû par un sincère et généreux désir de conciliation "... tout en invitant la fabrique à voter les fonds dont elle peut disposer. Le maire indique que, dans une quinzaine de jours, la commission d'examen aura à se prononcer sur les plans de l'architecte; il se plaît à croire que M. le curé sera le premier à engager le conseil de fabrique à désigner quelques-uns de ses membres pour en faire partie.

Toutefois, en dépit de la tentative de conciliation du maire, le différent avec le conseil de fabrique s'aggrave singulièrement. En témoigne éloquemment la longue lettre que le maire, visiblement excédé, adresse au sous-préfet le 8 juillet 1858, au sujet des difficultés suscitées par le curé qui n'a pas daigné répondre à sa lettre du 29 juin ${ }^{22}$.

" Je vous laisse apprécier s'il est désormais possible de marcher d'accord et d'arriver au but avec un conseil de fabrique composé comme l'est celui de Lambézellec, tout entier à la discrétion, à la merci d'un ou deux meneurs, dont toutes les dispositions ne tendent qu'à paralyser celles du conseil municipal et celles de l'Administration. "

Et le maire de se demander si toutes les démarches faites par le conseil municipal " avec autant de zèle que de générosité " vont être réduites à rien " devant la force d'inertie " qu'oppose le curé. Le comble de l'inconséquence est que, sur les onze membres du conseil de fabrique, six sont en même temps conseillers municipaux! Et le maire de révéler au sous-préfet les insinuations du curé.

"Le dimanche du Bon Pasteur [18 avril] ne s'est-il pas avisé de faire entendre du haut de la chaire de vérité, ces étranges paroles : "Priez, mes frères, priez avec ferveur [...]; priez, afin que le malin Esprit ne triomphe pas de nos projets : car je vous le dis, le malin Esprit travaille et s'agite pour en retarder l'exécution"... Il va sans dire, que, dans sa pensée, le malin Esprit, c'est l'Administration civile, c'est le conseil municipal, c'est vous et moi. Quelle conséquence ne pourrait avoir semblable doctrine, prêchée en langue bretonne, devant une population aussi profondément religieuse que celle de nos campagnes, qui n'admet pas, dans la bouche du prêtre, d'autre parole que la parole de $\mathrm{Dieu}^{23}$ !”

Le maire achève sa lettre en demandant au sous-préfet, " en cette circonstance de plus en plus épineuse ", de susciter un accord entre l'administration municipale et le conseil de fabrique.

Mais le différend ne paraît pas s'estomper. Dans une lettre du préfet à l'évêque de Quimper, en date du 10 décembre 1858, il est fait état d'un rapport du sous-préfet de Brest relatif au refus exprimé par le conseil de fabrique de modifier la délibération prise le 30 octobre au sujet des ressources à affecter pour la reconstruction de l'église ${ }^{24}$. Selon le préfet, "le

22. Id.

23. Et le maire d'ajouter : "Faut-il s'étonner, après cela, si les cultivateurs ne mettent plus autant d'empressement à transporter les moellons. "

24. À cette date, le conseil de fabrique avait pris l'engagement de contribuer à la dépense pour $26464 \mathrm{~F}$. 
refus du conseil de fabrique n'est qu'une nouvelle manifestation de l'opposition qu'il n'a cessé de faire à la reconstruction de l'église ". Comme cette opposition risque d'empêcher ou tout au moins de retarder la réalisation du projet - dont l'utilité ne peut être contestée - le préfet suggère à l'évêque de voir "s'il n'y aurait pas lieu de demander à M. le Ministre de l'Instruction publique et des Cultes, la dissolution du conseil de fabrique". Cette proposition était acceptée par l'évêque, ainsi que l'atteste une lettre du préfet du Finistère au ministre de l'Instruction publique et des Cultes, datée du 27 décembre 1858 : « Désespérant de vaincre une résistance de la fabrique (que rien n'explique), l'évêque de Quimper s'est vu forcé de demander la dissolution du conseil de fabrique. "Selon le préfet, il y a lieu de penser que le nouveau conseil de fabrique " secondera entièrement les efforts de l'administration municipale".

Le différend entre fabrique et municipalité était alors aplani. Lors de la séance du conseil de fabrique, le 25 septembre 1859, le curé, après avoir rappelé que le plan primitif de l'église projetée avait été modifié suite aux observations présentées par le ministre de l'Instruction publique et des Cultes, et le devis porté à environ $160000 \mathrm{~F}$, proposait au conseil de porter les fonds consacrés à la reconstruction de $26464 \mathrm{~F}$ à $28464 \mathrm{~F}$ - ce qui était voté à l'unanimité.

En fait, des problèmes entre fabrique et municipalité allaient encore surgir quelques années plus tard, lors de la question du dallage de l'église. Dans une lettre au préfet (24 janvier 1865) ${ }^{25}$, le sous-préfet, après avoir estimé, comme le maire, qu'il vaudrait mieux que le dallage soit fait dès à présent, ajoute qu'» il y a une limite à tout ». Et d'expliciter sa pensée :

"La commune de Lambézellec prend plus que sa part dans la reconstruction de l'église et... la fabrique fait le moins qu'elle peut. Ce sont ordinairement les fabriques qui concourent le plus largement dans la construction des édifices du culte. Il en a été tout autrement à Lambézellec. »

Après avoir rappelé qu'il a fallu dissoudre le conseil de fabrique pour triompher de sa résistance et en nommer un autre, le sous-préfet fait remarquer que, pendant que la commune de Lambézellec s'imposait " des sacrifices hors de toute proportion en comparaison de ceux de la fabrique, M. le curé faisait construire à Kerinou une chapelle qu'aucun besoin ne réclamait... La somme importante consacrée à cette chapelle eut été plus équitablement employée à la construction de l'église ». Le sous-préfet termine sa lettre en indiquant que " la fabrique de Lambézellec a de très beaux revenus et qu'il est temps qu'elle prenne dans la dépense de l'église une part plus en rapport avec ses ressources ". Dans ces conditions, elle peut fort bien prendre à son compte la dépense de $8997 \mathrm{~F}$ pour le dallage de l'église. Mais la fabrique qui a voté $5000 \mathrm{~F}$ pour le dallage, refuse d'en payer la totalité.

25. Arch. dép. du Finistère, 1 V 376. 


\section{Une adjudication difficile}

Le 29 juillet 1860, il est enfin procédé à une tentative d'adjudication des travaux de reconstruction ${ }^{26}$. M. Olivier Devilliers, entrepreneur à Lambézellec, offre de les exécuter, mais en demandant une augmentation des prix de base, fixés au devis, de $33 \%$, proposition évidemment refusée par la commission. Mais aucun autre entrepreneur ne répond à l'appel d'offre. Par ailleurs, une lettre du sous-préfet (29 août 1860) fait savoir que le sieur Devilliers ne présentant pas les garanties désirables ne peut, de toute façon, être chargé de l'entreprise. Dans ces conditions, que convientil de faire? L'architecte Bigot propose, le 6 septembre 1860, d'augmenter le prix du devis de $20 \%$ et de tenter une nouvelle adjudication. En fait, le devis, rédigé en 1858, avait été basé sur les prix pratiqués couramment à cette date, mais ceux-ci avaient rapidement augmenté, principalement en ce qui concerne la main-d'œuvre. Cette croissance brutale est à rechercher dans les immenses travaux publics entrepris - entre autres l'établissement du chemin de fer sur le tronçon Guingamp-Brest - qui rendaient les ouvriers exigeants ${ }^{27}$.

Conformément aux affiches placardées le 25 octobre 1860, une nouvelle tentative d'adjudication a lieu le 20 novembre. Plusieurs entrepreneurs se sont présentés, mais aucune offre n'est faite! Peu après, le 10 février 1861, le maire de Lambézellec annonce au conseil municipal qu'il a reçu deux propositions pour les travaux de l'église ${ }^{28}$. La première émane des " entrepreneurs de la chapelle des Jésuites à Brest ", qui demandent 27 \% d'augmentation sur le devis; par ailleurs, ils ne veulent pas se charger du clocher et, en outre, présentent diverses modifications qui, finalement, élèvent leurs prétentions à plus de $40 \%$ au-dessus du prix du devis; une telle exigence est rejetée à l'unanimité. La seconde proposition émane de M. M. Pérès frères qui acceptent toutes les clauses du devis et s'engagent à exécuter l'ensemble des travaux moyennant une augmentation de $32 \%$; toutefois, ils demandent une prolongation du délai d'achèvement d'exécution jusqu'au 31 décembre 1863. Le maire, tout en soulignant que ces conditions peuvent paraître bien dures à première vue, est toutefois d'avis de les accepter, d'autant plus que «M. M. Pérès offrent toutes les garanties désirables de responsabilité, d'aptitude et de moralité industrielle ". Mais quelques membres du conseil proposent que la décision soit ajournée à huitaine. Aussi le maire, après l'introduction de M. M. Pérès dans la salle des délibérations, leur demande huit jours de réflexion pour statuer sur leur offre. Les entrepreneurs répondent aussitôt qu'« ils ne veulent donner

26. Idem.

27. Le trop petit nombre d'ouvriers par rapport à la multiplicité des travaux les conduisait à se dire entre eux : "On ne peut se passer de nous... si on veut exiger de nous ce qui ne nous convient pas, eh bien, peu nous importe, d'autres chantiers nous attendent avec copieuses libations!!! » Pour plus de détails, se reporter à Louis CHAURIS, « La reconstruction de l'église Saint-Houardon ", Courrier du Léon/Progrès de Cornouaille, 8 janvier 1994, p. 18-19.

28. Arch. dép. du Finistère, 1 V 376. 
aucun délai; qu'ils ont fait, de leur côté, toutes les réflexions, et que si le conseil ne prend pas sa résolution séance tenante, ils se regardent instantanément comme déliés de tout engagement envers l'administration municipale ». Le maire, surpris et affligé par cette déclaration catégorique inattendue, soumet au vote la proposition des entrepreneurs qui est rejetée par 14 voix contre 9 .

Au début de l'année suivante, deux lettres du sous-préfet au préfet confirment la nécessité - mieux, l'urgence des travaux. Selon la première (19 mars 1862), « l'église de Lambézellec tombe littéralement en ruines. Elle ne peut plus servir ". Selon la seconde (8 avril 1862), "si on touche à une partie de l'église, tout va s'écrouler. Il n'y a qu'une chose à faire, c'est de hâter le commencement de la reconstruction. Le projet, les plans et le devis sont prêts et approuvés. Aussitôt l'indemnité due par Brest [pour l'annexion] fixée, on tentera l'entreprise des travaux ${ }^{29}$ ".

L'adjudication n'a toujours pas eu lieu! Toutefois, dans une lettre au préfet, en date du 29 novembre 1862, le sous-préfet estime que les circonstances sont à présent " très favorables " à une adjudication, par suite de la diminution notable, depuis deux mois, du prix des matériaux et de la maind'œuvre; en outre, plusieurs entreprises sont actuellement disponibles. Cependant le registre des délibérations de la mairie de Lambézellec, en date du $1^{\mathrm{er}}$ mars 1863, indique que la nouvelle tentative d'adjudication a encore échoué! Quatre entrepreneurs avaient fait des propositions, mais elles étaient tellement onéreuses qu'elles devenaient inacceptables.

Dans ces conditions, le devis estimatif est encore remanié : il s'élève maintenant à la somme de 246078,85 F. Le 7 avril 1863, le maire écrit à l'architecte qu'une nouvelle adjudication aura lieu le 23 de ce mois ${ }^{30}$. Il se montre optimiste sur l'issue de cette tentative : "Sans nul doute [écrit-il], la construction de la flèche étant ajournée, de nombreux concurrents se présenteront pour étudier... le devis des travaux à adjuger. " Effectivement, une lettre du sous-préfet de Brest, en date du 2 mai $1863^{31}$, fait connaître que l'adjudication a enfin abouti. Plus précisément, il s'agit de trois adjudications différentes : la première, consentie au sieur Gassis, moyennent une augmentation de $6 \%$, concerne la reconstruction de l'église proprement dite; la seconde, au sieur Lapierre, moyennant un rabais de $5 \%$, se rapporte aux travaux d'ornementation; la troisième, enfin, au sieur Viau, avec un rabais de $3,5 \%$, est relative à la fourniture de la chaux.

Ultérieurement, le 16 juin 1864, le maire fait savoir au sous-préfet que le conseil municipal désirerait voir également adjuger au sieur Gassis, entrepreneur de l'église, les travaux d'exécution de la flèche ${ }^{32}$ et la confection

29. La ville de Brest avait pris définitivement possession du territoire annexé à partir du 27 janvier 1862.

30. Arch. de l'évêché de Quimper (Lambézellec).

31. Arch. dép. du Finistère, 1 V 376.

32. En pierres œuvrées provenant des environs de Quimper. Arch. dép. du Finistère, $1 \mathrm{~V} 376$. 
du lambris - travaux que la commune s'était trouvée dans l'obligation d'ajourner en 1863 - ce qui permettrait de compléter l'édifice en cours d'exécution. Dans sa délibération du 12 juin, précédant de quelques jours l'envoi de la lettre du maire au sous-préfet, le conseil municipal avait en effet estimé qu'il était " dans l'intérêt de la commune de confier ces travaux à un entrepreneur habile, tel M. Gassis, dont l'Administration n'a qu'à se louer et qui conduit son entreprise avec... toute l'intelligence désirable ». M. Gassis s'était engagé à faire ces travaux au prix spécifié au devis, moyennant une augmentation de $6 \%$.

\section{Nature et provenance des pierres de construction}

L'une des questions majeures posées par tout édifice est, à l'évidence, celle de la nature et de la provenance des matériaux de construction, tant moellons que pierres de taille, éléments des mortiers (sable, chaux...), sans omettre les ardoises. Même dans une contrée au sous-sol aussi riche et varié que la Bretagne, les problèmes des approvisionnements en matériaux pierreux peuvent se révéler encore assez difficiles, dans la seconde moitié du XIX ${ }^{\mathrm{e}}$ siècle, d'autant plus que les qualités exigées pour les diverses parties de l'édifice s'avèrent différentes, et que les coûts des transports risquent d'alourdir fortement les budgets. Toutefois, comme souvent en Bretagne et singulièrement en Armor, où la mer est toute proche, les frais d'acheminement sont, à cette époque, sérieusement atténués par l'utilisation de la voie maritime. Parmi bien d'autres ${ }^{33}$, l'église de Lambézellec fournit un excellent exemple du rôle joué par la mer pour les approvisionnements en pierres de taille : granite de l'Aber-Ildut, extrait sur les rivages de l'Atlantique dans le Bas-Léon; kersanton en provenance des confins orientaux de la rade de Brest; granite des environs de Quimper. Mais il y a plus : une partie du sable est venue de l'estuaire de l'Aber-Ildut; les ardoises proviennent de la région de Châteaulin, via l'Aulne maritime et la rade de Brest. Par contre, les moellons, le plus souvent d'origine proximale, ont été transportés par charrois... bien qu'il semblerait que la Penfeld ait été également sollicitée ${ }^{34}$.

\section{Granite de l'Aber-Ildut}

Dans la seconde partie du XIX ${ }^{\mathrm{e}}$ siècle, le granite de l'Aber-Ildut - plus connu sous la dénomination de " Laber " - caractérisé par ses gros feldspaths roses et ses enclaves oblongues gris-noir, exploité intensément en bordure de la mer et sur les rives de la ria de ce nom ${ }^{35}$ était, avec le ker-

33. Voir, par exemple, CHAURIS, Louis, " Pierres de construction des églises du PetitTrégor ", Colloque Saint-Jean-du-Doigt, UBO-CRBC, Kreiz 14, p. 231-275.

34. Lettre du maire au curé de Lambézellec (29 juin 1858). Arch. dép. du Finistère, 1 V 376.

35. Chauris, Louis, "La saga des granites de l'Aber-lldut ", Courrier du Léon/Progrès de Cornouaille ( 8 et 15 juillet $-5,19$ et 26 août -16 septembre -21 octobre $-4,11$, 18 novembre -2 et 9 décembre $1995-6$ janvier 1996). 
santon, extrait le long des diverticules orientaux de la rade de Brest ${ }^{36}$, la pierre la plus estimée du Finistère. Toutefois, les deux roches ne présentent pas des aptitudes équivalentes ${ }^{37}$. C'est d'ailleurs ce que laisse clairement entendre l'architecte Bigot dans une lettre adressée le 20 mai 1863 au maire de Lambézellec ${ }^{38}$. L'entrepreneur Gassis avait informé l'architecte que le maire proposait d'élargir la porte principale de l'église, de supprimer le pilier central du porche et de substituer à la pierre de Laber celle de kersanton pour la porte principale. Aussitôt prévenu, l'architecte priait le maire de ne pas chercher à modifier la porte principale, ni en largeur, ni en nature de pierre. Et ce pour les raisons suivantes : "Le Laber est très résistant sous la charge du clocher, tandis que je n'aurai qu'une faible confiance [en] le kersanton dans cette substitution "... L'église de Lambézellec fournit aussi un exemple de la dureté du granite de l'AberIldut. En vue de loger une horloge dans la base du clocher, il avait été question d'effectuer en cet emplacement un trou de 0,50 m de côté ${ }^{39}$; mais l'architecte allait s'opposer à ce projet : " S'il faut couper un mur en pierre très dure de Laber, il est certain qu'un tel travail ébranlera et déconsolidera la base du clocher."

Si les qualités du granite de Laber sont unanimement reconnues, il n'en est pas moins vrai que son prix de revient reste élevé, surtout lorsque s'y adjoignent les frais d'acheminement. Ainsi s'interprète la démarche du maire de Lambézellec, adressant à l'architecte, le 15 avril 1863, un échantillon d'un autre " granite qui [lui] semble réunir de bonnes qualités ${ }^{40}$ "; ce granite a été extrait dans une carrière ouverte à environ $6 \mathrm{~km}$ du bourg de Lambézellec ${ }^{41}$. Le maire demande à l'architecte de "l'examiner attentivement et de [lui] faire connaître [son] opinion ». Et d'ajouter : "Si ce granite vous semble bon pour les travaux de gros œuvre et réunir les qualités que l'on donne à la pierre de Laber, il y aurait une économie considérable à l'employer quant à la distance et aux frais de transport. " Cette proposition ne semble pas avoir été retenue ${ }^{42}$. Par ailleurs, l'importance prise par le granite de l'Aber-Ildut, dans l'église de Lambézellec, est aussi soulignée,

36. CHAURIS, Louis, "Une pierre bretonne : le kersanton ", 119e Congrès national des sociétés historiques et scientifiques, Amiens 1994, Colloque CTHS "Carrières et constructions ", III, p. 279-296. Du même auteur, voir aussi : " Naguère... le kersanton et son emploi dans les travaux publics ", Le Mausolée, 1997, 726, p. 74-81; 727, p. 66-69; 728, p. 68-75.

37. Le kersanton est surtout connu pour son étonnante aptitude à la sculpture; le Laber pour sa résistance aux travaux à la mer...

38. Arch. de l'évêché de Quimper (Lambézellec).

39. Idem.

40. Id.

41. Selon toute probabilité, il s'agit d'un échantillon en provenance du grand massif granitique dit de Saint-Renan-Kersaint, qui affleure largement au Nord de Lambézellec. Se reporter à la carte géologique au 1/80000 , feuille "Brest " (partie Léon), 1972, levée par l'auteur.

42. Des données complémentaires sur l'emploi du granite de l'Aber-Ildut dans l'église de Lambézellec, seront présentées dans le chapitre consacré au coût de l'édifice. De même, pour le kersanton... 
quoiqu'indirectement, par le fait que, dans le devis, une erreur de $30000 \mathrm{~F}$, avait été commise dans l'estimations de ce matériau.

\section{Kersanton de la rade de brest}

Dans le projet de l'église, la flèche devait être initialement érigée en kersanton. Mais ledit projet devait être ultérieurement abandonné pour des raisons d'économie : il s'agissait de réduire, d'une manière significative, l'erreur commise sur le coût du granite de l'Aber-Ildut. À Lambézellec, le kersanton a été de préférence utilisé pour des travaux d'ornementation, confiés au sculpteur Lapierre ${ }^{43}$.

\section{Granite des environs de Quimper}

L'emploi du granite de la région quimpéroise pour la flèche de Lambézellec est dû à l'abandon du kersanton dans cette partie de l'église, en vue de réaliser, comme nous l'avons noté, de substantielles économies, suite à l'erreur de $30000 \mathrm{~F}$ commise dans l'estimation du granite de l'AberIldut. Mais le granite de Quimper - sans aucun doute moins célèbre que le kersanton - n'en a pas moins aussi ses titres de noblesse. Il est rappelé à cette occasion, lors de la délibération du conseil municipal du $1^{\mathrm{er}}$ mars 1863, que ledit granite a servi récemment pour la construction des flèches de la cathédrale de Quimper ${ }^{44}$; le granite du même district a été aussi utilisé pour l'église de Pont-Croix " dont la construction remonte à 400 ans et qui présente encore toutes les garanties possibles de solidité ${ }^{45}$ ». Quelques jours auparavant - le 24 février 1863 - avant que le conseil municipal ne prenne sa décision - le maire avait demandé à l'architecte de lui adresser " le plus tôt possible un échantillon de granite " de la contrée quimpéroise; il estimait, avec justesse, que sa proposition serait accueillie favorablement, du fait qu'elle se rapporte " aux intérêts bien entendus de la commune ». Aussi priait-il l'architecte de rédiger, avec « la plus grande célérité possible ", un nouveau devis pour la flèche.

\section{Moellons}

Les données archivistiques sont peu nombreuses sur la fourniture des moellons utilisés pour la reconstruction de l'église. Bien avant le début des travaux, la commune avait déjà entrepris d'accumuler des moellons. C'est ce qu'atteste une lettre du maire au curé, en date du 29 juin 1858 " Nous n'avons pas encore [écrit-il] la moitié des moellons nécessaires, et c'est avec beaucoup de peine que nous obtenons quelques voitures pour les enlever. Il est cependant bien essentiel que nous ne laissions pas passer

43. Henwood, Annie, "Le sculpteur Lapierre ", Cahiers de l'Iroise, 28, 1, 1981, p. 50-51.

44. Élevées - comme on l'a dit - sous la direction de J. Bigot (1854-1856). Voir BigOT, Joseph, "Les flèches de la cathédrale de Quimper ». Bull. Soc. archéol. Finistère t. X, 1883, p. 262-267.

45. Arch. dép. du Finistère, 1 V 376. 
une si belle occasion, puisqu'ils nous sont donnés gratis. " Ce document ne fournit aucune précision sur la nature et la provenance de ces moellons. Toutefois, à titre d'hypothèse, on peut présumer qu'ils étaient extraits dans l'arsenal de Brest où les travaux d'escarpement livraient d'énormes quantités de pierres qu'il fallait évacuer ${ }^{46}$. S'il en est bien ainsi, il s'agirait des " gneiss de Brest ", très recherchés comme moellons dans toute l'agglomération brestoise. Cette interprétation semble appuyée par une lettre du maire qui écrit, en parlant desdits moellons : "Je continuerai à les faire apporter à Penfeld " - village établi à l'extrême fond maritime de la ria de ce nom, dont le secteur aval abrite le port de guerre - et "à donner des hommes pour tenir le chenal libre".

Un autre document suggère, à son tour, l'appel aux gneiss de Brest. En l'occurrence, il s'agit d'une lettre du sous-préfet au préfet, datée du 29 novembre $1862{ }^{47}$. " La commune de Lambézellec a déjà approvisionné une certaine quantité de moellons. Elle compte, en outre, faire extraire de la carrière du Moulin à Poudre et transporter à pied d'œuvre tout le moellon qui sera nécessaire à la construction. " Cette importante carrière était ouverte dans les gneiss de Brest ${ }^{48}$.

\section{Ardoises}

Le devis avait prévu que la couverture de l'église serait effectuée en " ardoises très épaisses ${ }^{49}$ ". Dans une lettre à Gassis fils, l'architecte s'étonne que le père de l'entrepreneur, " habitant le pays d'ardoises [région de Châteaulin] ne puisse choisir sur les lieux tout ce qu'il y a de plus fort en échantillon ". En fait, le problème était bientôt résolu; Gassis fils faisait savoir à l'architecte (9 décembre 1864) qu'il avait " trouvé à Châteaulin une carrière dont les ardoises sont convenables ". Mais d'autres difficultés allaient surgir lors de l'exécution des travaux de couverture. Dans une lettre datée du 20 janvier 1865, Gassis fils se plaint, auprès de l'architecte, des couvreurs employés à la toiture. N'écrit-il pas : "Les couvreurs de ce pays sont de vrais ivrognes. " D'où l'interdiction, formulée à leur égard, de monter sur les toits " quand ils ne sont pas dans leur état normal, ce qui leur arrive assez fréquemment ".

46. ChAURIS, Louis, "La pierre dans le port militaire de Brest ", Bulletin de la société archéologique du Finistère, CXXXI, 2002, p. 237-275 et CXXXII, 2003, p. 253-274.

47. Arch. dép. du Finistère, 1 V 376.

48. Le " gneiss de Brest " est un ancien granite transformé par métamorphisme. Quand il est sain, il offre une teinte gris-bleuté; quand il est altéré - ce qui est la règle générale - il devient brunâtre par suite de l'oxydation de la pyrite disséminée. Son feuilletage plus ou moins prononcé le rend impropre à l'obtention des pierres de taille et son utilisation se limite à la confection de moellons.

49. Arch. de l'évêché de Quimper (Lambézellec). 


\section{Sables}

L'approvisionnement en sable pour la confection des mortiers paraît avoir soulevé quelques problèmes. Le 13 mars $1863^{50}$, le maire adresse à l'architecte un paquet contenant cinq échantillons de sable en provenance des carrières de Lambézellec - en l'occurrence, il s'agit très certainement d'arènes granitiques - dites aussi sable de mine. Il lui demande de les examiner et de voir " si l'un d'eux pourrait remplacer le sable de mer dont le transport serait excessivement coûteux ". Quelques jours plus tard, le 17 de ce mois, le maire demande à l'architecte d'apporter au cahier des charges et au devis, la modification suivante : « Il sera loisible à l'entrepreneur de faire usage du sable de Lambézellec, provenant des carrières qui avoisinent le bourg, sans obligation pour lui de faire usage de sable de mer. " Nous n'avons pu savoir si ledit sable de mine a été effectivement utilisé. Quoi qu'il en soit, le sable de mer semble avoir eu la préférence. C'est ce que laisse entendre une lettre de l'entrepreneur à l'architecte (9 mars 1865) ${ }^{51}$, l'informant qu'il est " assez difficile de se procurer du sable de Laber ${ }^{52}$ ", tous les bateaux étant retenus en ce moment " pour la fourniture du viaduc de Port-Launay ${ }^{53}$ ». En conséquence, l'entrepreneur estime qu'il sera obligé de payer le sable beaucoup plus cher, « pour la raison qu'il faudra fréter un bateau spécial pour aller en prendre ".

\section{Remploi de vieilles pierres}

Vu le coût des matériaux de construction et les difficultés des transports, le simple bon sens conduisait, en règle générale, à réutiliser - à remployer - les vieilles pierres provenant des démolitions. À Lambézellec, selon le premier devis, les matériaux provenant de la démolition de l'église, ainsi que de la chapelle Sainte-Anne, devaient être laissés à l'entrepreneur pour la somme de $6000 \mathrm{~F}$ Mais dans une lettre ${ }^{54}$, datée du 8 décembre 1862, le maire informe l'architecte que la commune a manifesté l'intention de conserver la chapelle " pour que le service religieux ne soit pas interrompu pendant les travaux de reconstruction de l'église ". Plus précisément, le maire demande que seuls soient démolis « le clocher et la façade de ladite chapelle construits en très belles pierres de taille ". Il n'est toutefois pas spécifié dans cette lettre si ces pierres seront employées dans la nouvelle

50. Idem.

51. Id.

52. CHAURIS, Louis, "L'extraction du sable à l'embouchure de l'Aber-Ildut ", Courrier du Léon/Progrès de Cornouaille du 7 novembre 1998. Voir aussi Arch. dép. du Finistère, $2 \mathrm{~S}$ 8, 4 S 56, 4 S 101.

53. Le viaduc de Port-Launay est dit aussi viaduc de l'Aulne. Tant par sa longueur totale (357 mètres, y compris les deux culées de $20,10 \mathrm{~m}$ ) et par sa hauteur maximale $(54,70 \mathrm{~m}$ des fondations au niveau des rails) que par l'élégance des 11 piles et peut-être surtout par l'ouverture des 12 arches en plein cintre $(22 \mathrm{~m})$, ce magnifique ouvrage d'art ne peut manquer d'éveiller la curiosité. Voir Arnoux, A., " Notice sur le viaduc de l'Aulne ", Paris, Dunod, 1870,56 p. 5 planches hors texte.

54. Arch. de l'évêché de Quimper. 
église ce qui est toutefois probable ${ }^{55}$. Cette interprétation semble confirmée ultérieurement par une lettre de l'entrepreneur à l'architecte (5 septembre 1863). Dans cette lettre, Gassis annonce qu'il a commencé la démolition de la chapelle Sainte-Anne, avec l'intention d'utiliser la pierre de taille qui s'y trouve et d'" occuper le plus grand nombre de tailleurs de pierre possible". Il constate toutefois que depuis l'adjudication, les carriers sont presque tous occupés par les travaux de la récolte des champs, si bien que les approvisionnements en pierres s'avèrent difficiles; de là, l'intérêt des remplois. Les autres pierres de démolition ne seront livrées à l'entrepreneur qu'à partir du moment où le service religieux sera établi dans la nouvelle église; dans ce dernier cas, il est évident que lesdites pierres ne seront pas remployées pour sa reconstruction.

Dans d'autres cas, le remploi est nettement attesté. Ainsi, lors de sa délibération du 13 novembre 1864, le conseil municipal avait voté, à l'unanimité, d'autoriser M. Gassis à utiliser dans les bas-côtés du nouvel édifice, les pierres de dallage de l'ancienne église et de la chapelle. De même une lettre de Gassis fils à J. Bigot ${ }^{56}$, datée du 11 mars 1865, indique un autre cas de remploi. Le curé avait demandé de daller une sacristie; mais l'architecte lui avait répondu que le dallage coûtait plus cher que le plancher... Or, sur ces entrefaites, Gassis fils annonçait à l'architecte qu'il venait " de découvrir, sous un dépôt de chaux, beaucoup de petites dalles carrées, d'environ $30 \mathrm{~cm}$ de côté " et qu'il ne savait qu'en faire. Aussi lui demandait-il de l'autoriser à les poser dans une des sacristies.

\section{Ressources financières et coût de la reconstruction}

L'église de Lambézellec a été reconstruite en 25 mois environ : la première pierre a été posée le 9 juillet 1863 et la consécration a eu lieu le 28 juillet 1865 .

Il a été noté qu'à la fin de 1859, le devis de la reconstruction était estimé approximativement à $160000 \mathrm{~F}$. Comment rassembler une telle somme? Il a été également indiqué que la commune a voté un emprunt de $100000 \mathrm{~F}$. Elle prélève, par ailleurs, sur ses revenus ordinaires, une somme de 5567,24 F. De son côté, comme cela a déjà été précisé, la fabrique a déclaré consacrer à la dépense la somme de $28464 \mathrm{~F}$. Ainsi, la somme totale disponible s'élève alors à $134031,24 \mathrm{~F}$; mais il manque encore 25968,76 F. Le préfet estime qu'il y a lieu d'accorder à la commune un secours de $20000 \mathrm{~F}$; suite à cette requête, le secours attribué ne sera alors que de $6000 \mathrm{~F}$, en trois annuités égales.

Le conseil municipal s'était depuis longtemps soucié des problèmes financiers soulevés par la reconstruction projetée. Ayant appris que la venue à Brest de l'Empereur était considérée comme certaine dans un avenir prochain, le maire de Lambézellec, lors de la séance du conseil muni-

55. Ou dans une autre construction de l'entrepreneur.

56. Arch. dép. du Finistère, 1 V 376. 
cipal du 9 mai 1858, revenant sur la question de la reconstruction de l'église, croit que rien ne serait plus propre à faciliter la solution de ce problème que de profiter de la présence de Sa Majesté pour la prier de venir poser la première pierre. Mais encore faudrait-il que les fondations de l'édifice soient amorcées... Aussi le maire demande-t-il à son conseil l'autorisation d'exécuter, par anticipation, les dépenses en vue de l'ouverture d'une tranchée de fondation, "seulement sur l'espace strictement nécessaire pour l'opération, en attendant que toutes les formalités aient été remplies ". L'opinion du maire est partagée unanimement par le conseil municipal ${ }^{57}$.

Vers la fin du même mois de mai 1858, le maire écrit au préfet pour lui exposer les motifs qui ont conduit le conseil municipal à cette proposition.

"C'est que cet acte de sa Majesté produirait sur nos populations bretonnes si religieuses, un effet magique et aurait dans le pays un retentissement immense pour le présent et pour l'avenir. Quelle gloire pour nos bons habitants de Lambézellec de pouvoir se dire et de faire répéter par les générations futures : "La première pierre de cette église a été posée par l'Empereur Napoléon III! C'est le premier et le seul souverain qui ait honoré notre pays de sa présence!" "

Le maire ajoute qu'une telle visite serait l'occasion inespérée d'aplanir les difficultés rencontrées par le projet et, en même temps - et c'est sans doute son intérêt majeur - " une garantie certaine de secours que le gouvernement de l'Empereur sera disposé à nous accorder ultérieurement et un motif d'espérer qu'il concourrait à la dépense dans une plus large proportion ". En un mot, cette visite serait " un pas immense vers la réalisation d'un projet qui s'agite depuis plus de trente ans "... Ce rêve ne devait pas se réaliser.

Mais un autre événement allait fournir à la commune une forte somme d'argent. Suite à l'annexion d'une partie de Lambézellec par la ville de Brest, le contingent de cette dernière cité à la reconstruction de l'église avait été fixé par l'Administration supérieure à 80000 F. Dans sa séance du 21 octobre 1862, le conseil de fabrique se réjouit d'apprendre que la commune va enfin pouvoir commencer " une construction si longtemps désirée et si impatiemment attendue ". Il évoque "l'heureux moment où va enfin cesser la situation vraiment triste et intolérable dans laquelle se trouve la population de Lambézellec pour l'accomplissement de ses devoirs religieux ", et, mieux, vote alors, à l'unanimité, une somme se montant globalement à $40000 \mathrm{~F}$.

Divers documents permettent de suivre l'impressionnante progression des coûts de la reconstruction. Prévue en 1855 pour $124789,95 \mathrm{~F}$, la dépense présumée s'élève à $156467,69 \mathrm{~F}$ en 1858 , puis en 1862 , à $230000 \mathrm{~F}$. À la fin des travaux, la reconstruction atteindra la somme colossale de $314205,62 \mathrm{~F}^{58}$. On comprend ainsi aisément que même après l'achèvement

57. Idem.

58. L'église de Douarnenez a atteint un coût assez comparable. Ces sommes paraissent singulièrement élevées quand on les rapproche de celles des grands ouvrages d'art exécutés à la même époque. Ainsi $2674540 \mathrm{~F}$ pour le gigantesque viaduc de Morlaix (1861-1863). 
de l'édifice, les problèmes financiers sont encore loin d'être tous résolus. C'est ce que précise une lettre du préfet en date du 19 février 1868. Sur la somme indiquée ci-dessus, la commune doit encore 82960,81 F. Dans ces conditions, le préfet insiste de la manière la plus pressante pour que l'Administration supérieure veuille bien accorder un secours supplémentaire et propose de fixer ledit secours à 10000 F. En fait, le 18 mai 1868, le ministère devait offrir un montant de $5000 \mathrm{~F}$.

Dans un rapport, daté du 12 mai $1866^{59}$, l'architecte Bigot expose en détail les causes de la différence entre l'estimation du devis de la reconstruction et le décompte définitif des travaux. En fait, il s'agit principalement des problèmes posés par les fondations de l'édifice, la profondeur du substratum solide s'étant avérée loin d'être en rapport avec les prévisions. Des sondages, entrepris avant l'établissement du projet, avaient rencontré un terrain ferme à une profondeur moyenne de 1,50 m, qui avait été estimée, à tort pour être constante. Or, en cours d'exécution, les fouilles est et ouest de l'église, à partir du mur nord du transept jusqu'à l'abside, ont dû être conduites à une profondeur moyenne de $3,20 \mathrm{~m}$, soit plus du double prévu. Plus grave, pour le mur ouest du transept, une fouille effectuée à plus de 4,50 m de profondeur, ayant rencontré un sous-sol mou et humide, il a fallu employer une assez grande quantité de béton. De même, pour les fondations du clocher, si une partie des fouilles a rencontré la roche, une autre partie a traversé un terrain dépourvu de résistance, d'où s'échappait une source. Pour prévenir d'éventuels déchirements dans la maçonnerie, il a été nécessaire de faire appel au béton et à des blocs de pierre de taille. Et c'est ainsi que " les fondations ont... dépassé la moitié de la dépense prévue au devis".

Par ailleurs, les maçonneries des fondations devaient être faites au mortier de chaux hydraulique et de sable. Par suite de la très forte augmentation du cube des fondations que nous venons d'indiquer, la fourniture de chaux prévue au devis s'est avérée insuffisante, d'où la nécessité d'achat complémentaire. Comble de malchance, la chaux hydraulique commandée avait dû être refusée et rebutée. L'absence de bonne chaux hydraulique dans la région brestoise, jointe à l'approche de l'hiver, laissant craindre des éboulements dans les fouilles, l'entrepreneur a été dans l'obligation d'employer la chaux de Brest, mêlée au ciment de Portland, d'où augmentation de la dépense. Il était initialement prévu d'exécuter le soubassement du clocher en chaux ordinaire au-dessus du sol; mais du fait de l'hétérogénéité des fondations en ce point, on a dû employer " parmi la chaux une certaine quantité de ciment afin d'ajouter à l'adhérence et à la prompte dessiccation des mortiers ». Enfin, la plus grande profondeur des fondations a exigé naturellement une base d'empâtement nettement plus large que celle prévue initialement.

59. Arch. de l'évêché de Quimper (Lambézellec). 
Le volume de la pierre de taille a été sensiblement dépassé pour deux raisons. La première est due au fait que, contrairement au devis, le conseil municipal a demandé que les remblais prévus sur la place de l'église jusqu'à une certaine hauteur, ne soient pas exécutés, ce qui a entraîné l'édification d'un socle sur toute la hauteur de la différence du niveau de la place. La seconde est liée à la présence d'un terrain inégal et douteux en certains points - ce qui conduit à donner aux pierres de très fortes dimensions, en particulier dans les contreforts ${ }^{60}$.

Il deviendrait bien vite fastidieux d'énumérer le détail des prix de la reconstruction de l'église. Aussi nous bornerons-nous à présenter quelques informations d'ensemble sous forme de tableaux, éventuellement complétés par un commentaire.

\section{Liste des différentes pierres utilisées pour l'église de Lambézellec} et de leur coût respectif ${ }^{61}$

\begin{tabular}{|l|c|c|c|}
\hline & Cubage/superficie & Prix de l'unité & Total \\
\hline Volume de Laber & $1074,28 \mathrm{~m}^{3}$ & $55 \mathrm{~F}$ & $59085,40 \mathrm{~F}$ \\
\hline Volume de kersanton & $210,52 \mathrm{~m}^{3}$ & $65 \mathrm{~F}$ & $13683,80 \mathrm{~F}$ \\
\hline $\begin{array}{l}\text { Superficie à moulures } \\
\text { de kersanton }\end{array}$ & $858,83 \mathrm{~m}^{2}$ & $20 \mathrm{~F}$ & $17176,60 \mathrm{~F}$ \\
\hline Superficie à moulures de Laber & $593,04 \mathrm{~m}^{2}$ & $20 \mathrm{~F}$ & $11860,00 \mathrm{~F}$ \\
\hline Dallage en kersanton et Laber & $875,98 \mathrm{~m}^{2}$ & $18 \mathrm{~F}$ & $15767,64 \mathrm{~F}$ \\
\hline $\begin{array}{l}\text { Flèche principale à partir } \\
\text { de la dernière galerie, } \\
\text { en pierre de taille de Quimper }\end{array}$ & $51 \mathrm{~m}^{3}$ & & \\
\hline
\end{tabular}

Le cubage en granite de l'Aber-Ildut est sensiblement cinq fois supérieur à celui du kersanton. Le prix $\left(\mathrm{m}^{3}\right)$ du kersanton $(65 \mathrm{~F})$ est supérieur à celui du Laber (55 F) et du granite de Quimper (60 F).

\section{Décompte de la fourniture de chaux et de ciment (entrepreneur Viau)}

Chaux hydraulique (69 hectolitres à $25 \mathrm{~F}$ l'un)

60. Une autre cause de l'augmentation de la dépense provient de la différence entre le métré réel des ouvrages effectués et les prévisions portées au devis.

61. Arch. de l'évêché de Quimper (Lambézellec). 
Le présent décompte, dressé par l'architecte J. Bigot, était reconnu et accepté par le fournisseur le 31 janvier 1867.

Dans une lettre du 13 novembre 1863, le maire avait informé l'architecte $^{62}$ que l'entrepreneur lui avait fait savoir que les travaux pouvaient être à présent poursuivis sans employer le ciment de Portland, qu'il serait possible de remplacer par la chaux grasse ordinaire. Et d'ajouter : "Veuillez me faire connaître si telle est aussi votre opinion. Dans ce cas, la commune trouverait un grand avantage, au point de vue de la dépense.

Les travaux d'ornementation en kersanton avaient été exécutés par le sculpteur Lapierre, qui transmettait son compte à l'architecte le 13 octobre $1865^{63}$. À cette occasion, il écrivait : "Vous n'avez pas oublié qu'il s'est trouvé deux gargouilles de trop. Je ne les porte pas au compte, quoique j'ai en main une note signée de vous qui a été la cause de cette erreur... Vous ferez à ce sujet ce que bon vous semblera. " Dans la même lettre, Lapierre demande de faire solder son compte promptement, car, ajoute-il : " J'ai grand besoin de fonds. " Près de deux ans après (le 5 juillet 1867), le même Lapierre écrivait à Bigot : "Les travaux ne vont pas du tout... aussi seraisje heureux si, à l'occasion, vous vouliez bien vous souvenir de moi et me confier l'exécution d'une partie de vos travaux. "

La délibération du conseil municipal du 24 novembre $1867^{64}$ précise que " la dépense totale des travaux d'ornementation en pierre de kersanton exécutés par M. Lapierre... dans la nouvelle église, doit être définitivement fixée à $11028 \mathrm{~F}$ ", d'où est déduit le rabais de $5 \%$ (551,40 F), soit 10476,60 F Lapierre ayant déjà reçu $9500 \mathrm{~F}$, il reste encore à lui payer 976,60 F.

Le coût des moellons est évidemment, très inférieur à celui de la pierre de taille. Le 8 décembre 1862, le maire écrit à l'architecte que, "d'après les renseignements qui [lui] ont été adressés par les propriétaires des carrières des environs, il faut compter en moyenne sur le prix de 3,50 F à $4 \mathrm{~F}$ le mètre cube... de moellons rendus à pied d'œuvre ". Nous ne nous étendrons pas plus longuement sur le coût des matériaux, des transports et de la maind'œuvre. Évoquons seulement encore deux informations. Les travaux supplémentaires exécutés à la tour de l'église sont facturés, le 4 janvier 1866, de la manière suivante à $\mathrm{M}$. Le Naour ${ }^{65}$ : 48 aigrettes à $3 \mathrm{~F}$ l'une (144 F); 7 boulons en kersanton pour relier les assises supérieures et percements des trous dans les assises à $4 \mathrm{~F}$ l'un (28 F)... Le transport de tous les matériaux nécessaires à la construction de la flèche et de ses accessoires, depuis Quimper jusqu'à Lambézellec, s'est levé - comme estimé au devis - à $2000 \mathrm{~F}^{66}$.

\section{Idem.}

63. Id.

64. Arch. dép. du Finistère, 1 V 376.

65. OGĖs, Louis, "Le Naour, le bâtisseur de clochers ", Bulletin de la société archéologique du Finistère, t. XC, 1964, p. XXVIII et LXIV-LXVI.

66. Décompte des travaux par Bigot, 29 novembre 1866. Arch. de l'évêché de Quimper (Lambézellec). 
La longue analyse à laquelle nous nous sommes livré est apparue indispensable pour révéler, dans leur diversité et leur complexité, les problèmes soulevés par l'exécution d'un édifice religieux, à une époque - le second Empire - où les constructions, et en particulier les travaux publics, ont atteint, dans un laps de temps très court, en Bretagne, une ampleur jamais égalée. Que l'on songe à l'établissement des chemins de fer à cette période, mais aussi aux nombreux forts ${ }^{67}$ échelonnés le long du littoral, aux phares ${ }^{68}$ et aux infrastructures portuaires...

Très schématiquement, l'étude de la reconstruction de l'église de Lambézellec conduit aux conclusions suivantes. En premier lieu, c'est une œuvre de longue haleine, non pas tant par son exécution proprement dite qui, en fait, a été rapide (en un peu plus de deux ans seulement), que par les multiples tergiversations entre les premiers souhaits et l'achèvement du bâtiment. Cette longue durée est en grande partie due au fait qu'il s'agit d'une œuvre collective, mettant en jeu non seulement le conseil municipal, singulièrement le maire - dont on ne saurait trop souligner ici le rôle majeur ${ }^{69}$ - et le conseil de fabrique (avec le curé), mais aussi l'architecte, les entrepreneurs, la population communale pour des charrois... De là des heurts inévitables. En particulier, la mauvaise volonté - difficile à comprendre - du conseil de fabrique, va même aller jusqu'à entraîner sa dissolution. En un mot, se déroulent ici les diverses péripéties d'une aventure humaine - avec ses travers et ses impondérables - même si elle a pour finalité le culte de la Divinité.

La construction de cette vaste église s'avère aussi une œuvre coûteuse, soulevant de complexes problèmes financiers. En fait, il apparaît que ce n'est pas tant le manque absolu de ressources qui pose des interrogations que les difficultés rencontrées pour les réaliser. De là, la nécessité de faire appel à de multiples financements (fonds disponibles, emprunts, secours de l'État...). À l'époque de l'établissement des devis de Lambézellec, la conjoncture s'est montrée peu favorable, avec la croissance rapide des coûts de la main-d'œuvre et des matériaux, liée à l'exécution des grands travaux dans toute la région. Ainsi s'explique, en partie, le fait qu'entre le devis initial et le prix de revient final de l'ouvrage, la somme totale ait été multipliée par plus de deux fois. À cette conjoncture indépendante de la volonté des acteurs, sont venus s'ajouter des imprévus lors de l'exécution, en particulier les problèmes soulevés par les fondations. On peut s'étonner que l'architecte se soit fié aux sondages préliminaires qui donnaient une profondeur moyenne de 1,50 m pour lesdites fondations, alors que l'expérience prouve que l'altération du sol est, en principe, irrégulière et aléatoire ${ }^{70}$.

67. Réduits, " modèle " 1846.

68. CHAuRIs, Louis, "Des carrières aux tours. La construction des phares en Bretagne occidentale ", 119e Congrès national des sociétés historiques et scientifiques, Amiens, 1994, Colloque CTHS, Carrières et constructions III, p. 297-315.

69 . Entre autres, dans ses efforts pour abaisser au maximum les frais de la construction.

70. Ainsi, lors de la construction du phare de Lanvaon à Plouguerneau, sous le Second Empire (Arch. dép. du Finistère, 4 S 1246). D'après l'aspect du terrain en surface, l'ingénieur pensait s'appuyer sur le rocher " en déblayant seulement une faible épaisseur de 
L'église de Lambézellec est typiquement une œuvre polylithique, c'està-dire ayant mis en jeu des pierres de nature et de provenance différentes. On retrouve, sans surprise, dans le bâti, les matériaux les plus utilisés au cours de la seconde partie du XIX ${ }^{\mathrm{e}}$ siècle dans le Bas-Léon, en particulier le granite de l'Aber-Ildut (le Laber), en association avec le kersanton de la rade de Brest ${ }^{71}$. L'emploi du granite, nettement plus distal, de Quimper, est plus curieux. Il s'interprète à la fois par des raisons d'économie et aussi par l'appel à l'entrepreneur Le Naour, spécialisé dans la fourniture des matériaux pour les clochers ${ }^{72}$. L'utilisation des ardoises de Châteaulin s'explique facilement : ce district était alors le principal centre d'extraction du Finistère; en outre, la production était aisément acheminée par voie d'eau à partir de Port-Launay.

Comme la plupart des églises reconstruites dans le Finistère sous le Second Empire, Lambézellec n'offre guère d'originalité architecturale. À cette époque, les concepteurs reproduisaient, sans se lasser, avec quelques variantes seulement, les modèles gothiques élevés avec tant d'élégance dans des siècles lointains. Tout se passe comme si la veine artistique était alors tarie. Comme bien d'autres, l'église de Lambézellec est une œuvre pastiche dans le style ogival du $\mathrm{Xv}^{\mathrm{e}}$ siècle. Ce constat n'implique pas son absence d'intérêt. Simplement, il ne faut pas y rechercher une inspiration créatrice.

terre végétale ". Mais les apparences étaient trompeuses, si bien que pour atteindre le rocher compact, "il a fallu descendre à des profondeurs qui ont dépassé 5 mètres en quelques points ".

71. Cette association est extrêmement fréquente dans la région brestoise. Les grands entrepreneurs locaux (Omnès, Corre) possédaient des carrières dans les deux districts et faisaient constamment appel à ces deux matériaux, tant pour l'habitat que pour les travaux publics.

72. À titre d'exemple, parmi d'autres : lors de la reconstruction de l'église de Carantec (Finistère) sous le Second Empire, avaient été utilisés les granites de l'île Callot, de l'île Grande, de Cléder et de Guerlesquin. Mais pour la flèche, érigée ultérieurement, appel avait été fait à Le Naour; la pierre était acheminée par voie d'eau, à partir de Quimper. Pour plus de détails, voir CHAURIS, Louis, "Pierres de Carantec, I, Monuments religieux ", Éd. presbytère de Carantec, 1996, 60 p. 
La reconstruction de l'église de Lambézellec sous le Second Empire

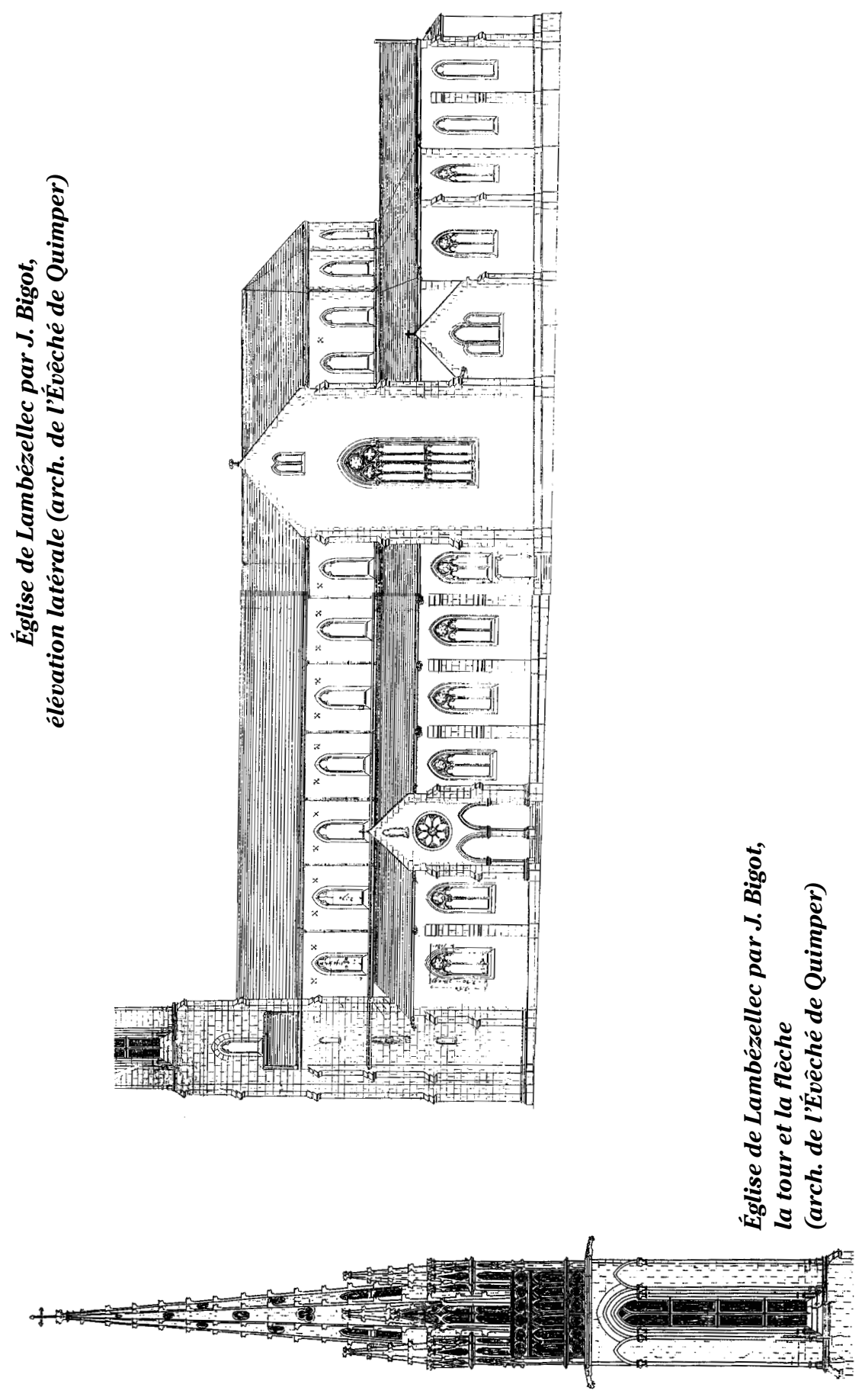




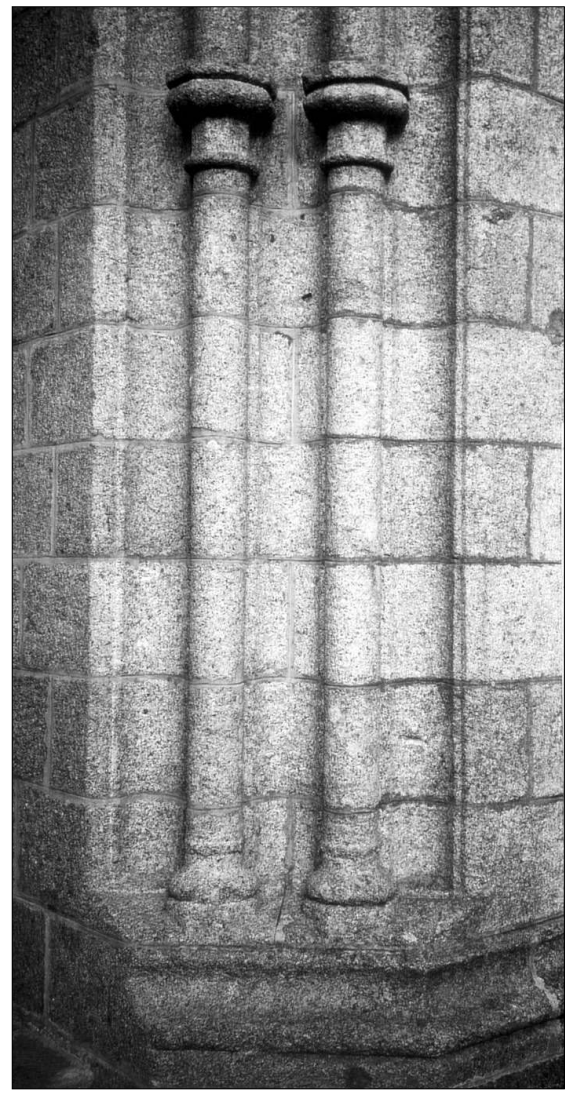

Colonnettes engagées du porche principal, sous le clocher, en granite à feldspaths roses de l'Aber-Ildut. Régularité des assises en pierre de taille

Porche latéral (gauche) en granite de Laber. Les répara-

tions effectuées après la guerre avec du ciment grisâtre, miment curieusement les sombres enclaves du granite. Seuls les chapiteaux sont en kersanton gris

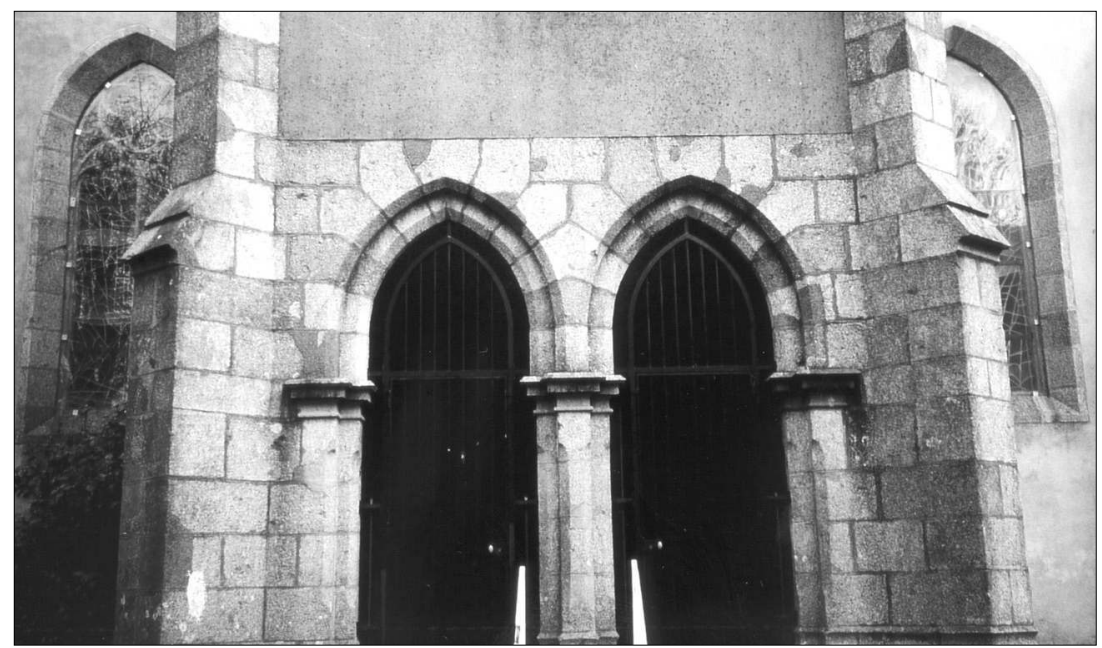




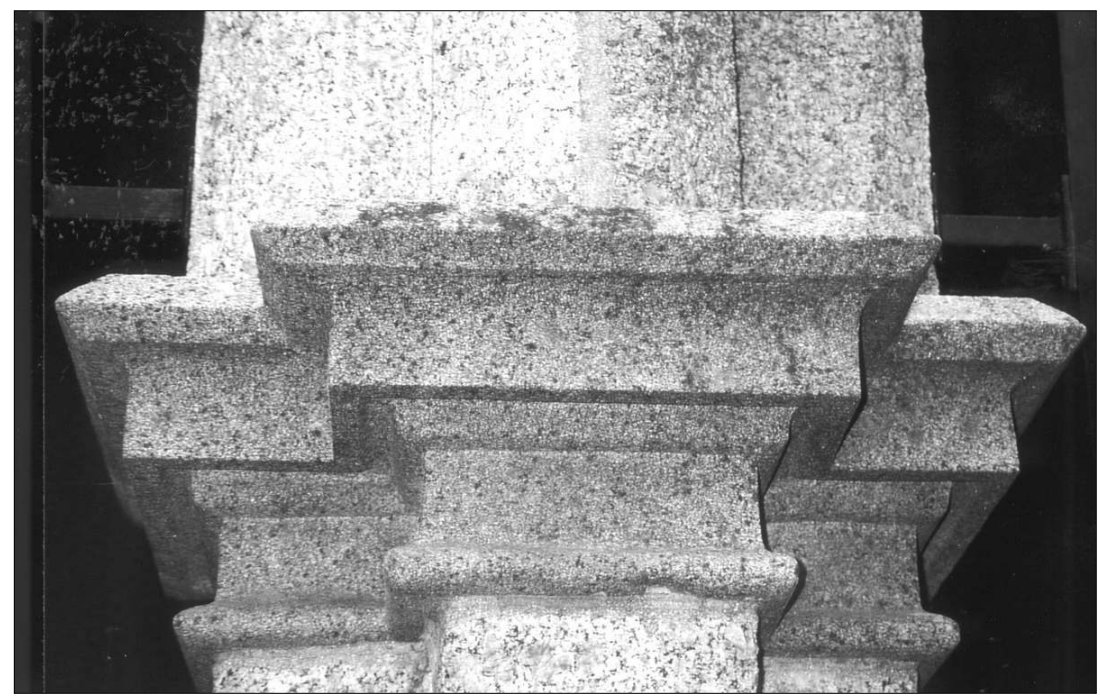

Détail du porche latéral (droit). Chapiteau en kersanton gris de part et d'autre du granite rose de Laber

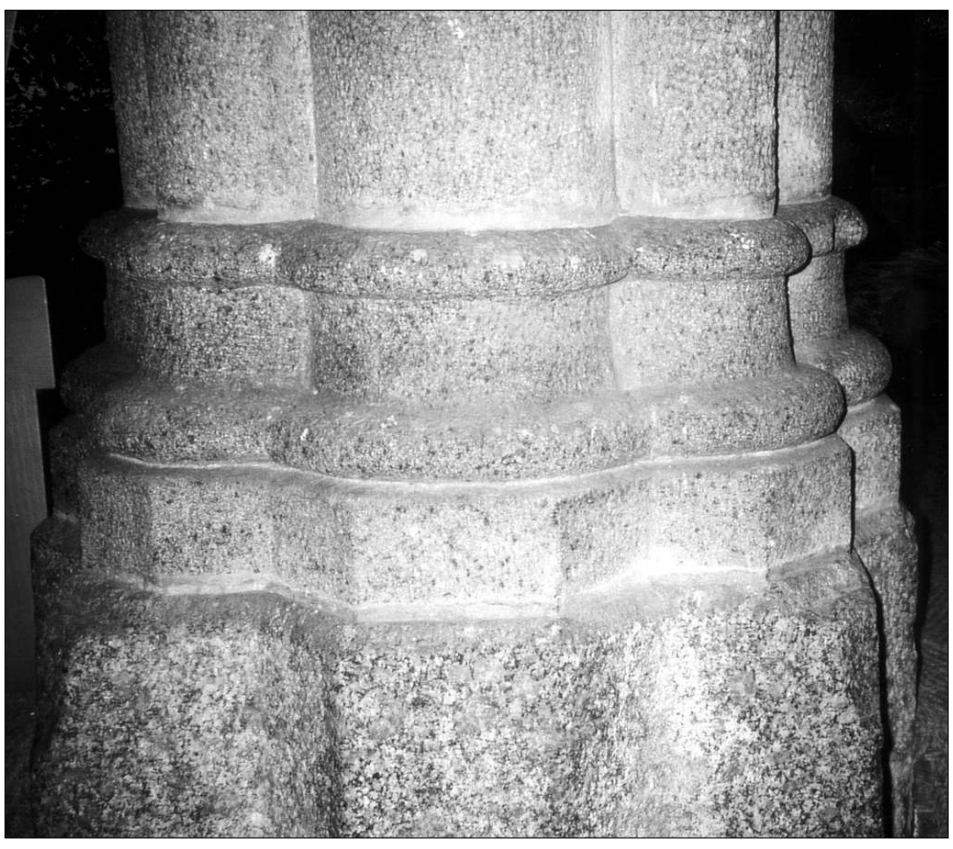

Partie inférieure d'un pilier.

Assise basale en granite porphyroüde rose de l'Aber-Ildut, surmontée par du kersanton gris sombre avec moulurations 


\section{RÉSUMÉ}

L'église de Lambézellec (Finistère) fournit un exemple instructif de reconstruction d'un édifice religieux sous le Second Empire. Après l'abandon d'un projet de restauration, jugé inacceptable vu l'état de vétusté et la dimension exiguë de la vieille église, est élaboré le plan de reconstruction d'un vaste édifice. La controverse avec le conseil municipal conduit à la dissolution du conseil de fabrique, impliqués l'un et l'autre dans le projet. Plusieurs tentatives d'adjudication échouent, entraînant une augmentation considérable des prix. Appel est fait à diverses sources de financement : fonds disponibles, emprunts, secours...; il sera même envisagé de demander à Napoléon III de poser la première pierre, en espérant ainsi une aide substantielle. Finalement, le coût de l'ouvrage sera multiplié par plus de deux fois, par rapport au devis initial. Construit sur les plans de l'architecte Bigot, par l'entrepreneur Gassis, avec la collaboration du sculpteur Lapierre, l'édifice est typiquement polylithique : granite de l'Aber-Ildut extrait en bordure de l'Atlantique pour la pierre de taille, kersanton dans les confins orientaux de la rade de Brest, plus particulièrement pour les travaux d'ornementation, granite de la région quimpéroise pour la flèche, ardoises du bassin de Châteaulin pour la toiture, gneiss locaux pour les moellons, remploi de vieilles pierres. Il se présente comme un pastiche, dans le style ogival du $\mathrm{Xv}^{\mathrm{e}}$ siècle, dépourvu d'originalité architecturale, et, au total, apparaît comme la manifestation d'une aventure humaine, avec ses heurts et ses avatars, même s'il a pour finalité le culte de la Divinité.

\section{ABSTRACT}

Rebuilding of a church during the second Empire: Lambézellec (Finistère). The church of Lambézellec provides a good example of the rebuilding of an edifice during the second Empire, after the renunciation of a restoration. Several attempts of adjudication break down, producing a strong increase of cost (above twice the starting price). The church is typically polylithical: Aber-Ildut granite near Atlantic ocean for building-stones; kersantite from the Brest roads for ornementation; granite from Quimper area for the spire; slates of Châteaulin basin for roofing; local gneiss for quarry-stones... The edifice is an imitation, in the gothic style, without architectural originality; it appears as the manifestation of a human experience, with nevertheless for final purpose the divine worship. 